\title{
Outage Probability and BER of the Ground to Train Communication Link of a Curved Track in Raining Turbulence with Pointing Errors
}

\author{
Yanqiu $Y A N G^{1}$, Yixin $Z H A N G^{1,2}$, Lin $Y U^{1,2}$ \\ ${ }^{1}$ School of Science, Jiangnan University, 214122 Wuxi,China \\ 2 Jiangsu Provincial Research Center of Light Industrial Optoelectronic Engineering and Technology, 214122 Wuxi, China \\ riely79@163.com,zyx@jiangnan.edu.cn,yulin@jiangnan.edu.cn
}

Submitted January 26, 2017 / Accepted April 9, 2017

\begin{abstract}
We model the outage probability and bit-error rate (BER) for an intensity-modulation/direct detection optical wireless communication (OWC) systems for the groundto-train of the curved track in rainy weather. By adopting the inverse Gaussian models of the raining turbulence, we derive the outage probability and average BER expression for the channel with pointing errors. The numerical analysis reveals that the rainfall can disrupt the stability and accuracy of the system, especially the rainstorm weather. The improving of the shockproof performance of the tracks and using long wavelength of the signal source will improve the communication performance of OWC links. The atmospheric turbulence has greater impact on the OWC link than the cover track length. The pointing errors caused by beam wander or train vibration are the dominant factors decreasing the performance of OWC link for the train along the curved track. We can choose the size of communication transmitting and receiving apertures to optimize the performance of the OWC link.
\end{abstract}

\section{Keywords}

Ground-to-train, curved track, inverse Gaussian model, optical wireless communications, raining turbulence

\section{Introduction}

Recently, OWC has increasingly attracted much attention for the last mile access environment due to its merits such as the large bandwidth, low cost, no licensing requirements, and high security [1-3]. OWC also can be adopted in order to offer higher data rates and provide seamless data services to the passengers traveling by train, buses, and ships [4], thereby increasing the demand of high-speed wireless access under the moving conditions. Paudel et al. [4] has proposed model of OWC link for ground-to-train where the OWC link only for the straight track in clear weather. However in the move of a train along the track, the train is vibrating along or perpendicular to the track. In this study, they did not consider the pointing errors between the transmitter and receiver. In addition, the ground-to-train OWC system works around the clock, and it may meet the extreme weather such as haze, fog, rain, and snow. The atmospheric turbulence can result in the refractive index fluctuations, signal scintillation, beam broadening, and beam wander [5]. All of these can affect the performance of the OWC channel. Taking account of the pointing errors and atmospheric turbulence, by using the gamma-gamma distribution, Gao et al. [6] established a model of the average capacity of the ground-to-train OWC link in fog-haze weather and pointed out that the pointing errors between the transmitter laser and receiver detector are dominant factor to decrease the average capacity of links.

As we know, the train must pass along the curved track except the straight track. Recently, Paudel et al. [7] has expanded model of OWC link along the straight track to curved track. Subsequently, Yang et al. [8] modeled the average capacity of the ground-to-train OWC link of a curved track with pointing errors, considering the atmospheric turbulence and fog-haze weather. However, rainfall must occur in nature. The model of OWC links in fog and clear weather has been established, but the model in rainy weather has not. Considering the distribution of rainfall-induced irradiance fluctuations can be modeled by inverse Gaussian model [9], [10], then the Gamma-Gamma distribution can be approximated by the inverse Gaussian model. Moreover, pointing errors and atmospheric turbulence occur at the same time, and can jointly increase the BER and outage probability [11], which are the main performance indicators of the system and has not been discussed for ground-to-train OWC link. In this paper, we use the inverse Gaussian models to characterize the rain-turbulent-induced irradiance fluctuation of the signal, then model outage probability and BER of the OWC link of the ground-to-train for the train along the curved track. We analyzed the effects of rainfall intensity, the turbulenceinduced beam wander and spread, curvature radius of the track, and the pointing errors on the performance of the link under raining condition. 
In this paper, we describe the geometrical model of the ground-to-train OWC link of the curved track in Sec. 2. In Sec. 3, we discuss the optical channel fading model and derive the expressions of outage probability and average BER in rainy weather. In Sec. 4, numerical results and analysis of the various parameters' effects are presented. Finally, We end with conclusions in Sec. 5.

\section{Geometrical Model of Curved Track}

Considering a OWC link for the ground-to-train of a curved track with the atmospheric turbulence in rainy weather, the geometrical model of the system is shown in Fig. 1 [8]. The optical transmitter (TX) positions on the ground stations alongside the track and optical receiver $(\mathrm{RX})$ positions on the roof of the train. The spacing between neighboring stations is required to make the beam fully cover the entire train. The corresponding stations are active, only when the train is in the transmission range.

The transmission length along the TX to $\mathrm{RX} z$ can be expressed by [8]

$$
z=\sqrt{R^{2} \sin ^{2} \omega+\left[d_{1}+R(\cos \omega-1)\right]^{2}}
$$

where $R$ is the curvature radius, $L$ is the covered track length, $\omega=\left(L+d_{2}\right) / R$ stands for the intersection angle of the center of the curve and points of RX and TX, $d_{1}$ is the distance between the base station and the track of the train, and $d_{2}$ is the distance of the $\mathrm{C}$ from the point ST.

The beam divergence of the transmitter $\theta$ is given by [8]

$$
\theta=\alpha+\beta=\tan ^{-1}\left(\frac{d_{1}}{d_{2}}\right)+\tan ^{-1}\left[\frac{d_{1}+R(\cos \omega-1)}{R \sin \omega}\right]
$$

where $\alpha$ and $\beta$ stand for the angle of the coverage beam at the longest point RX and the shortest point ST, respectively.

The radial offset $r$ from the axis $\mathrm{X}$ at the point $\mathrm{B}$ can be written as

$$
r=z \sin \left\{\frac{1}{2}\left[\tan ^{-1}\left(\frac{d_{1}}{d_{2}}\right)+\tan ^{-1}\left(\frac{d_{1}+R \cos \omega-R}{R \sin \omega}\right)\right]\right\} .
$$

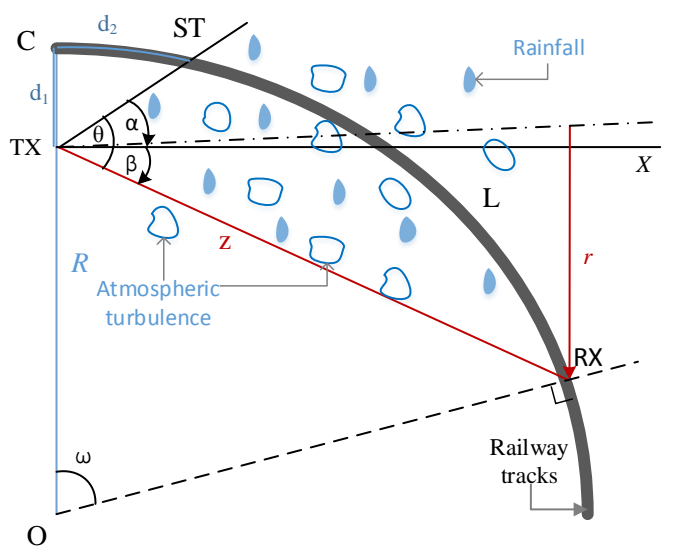

Fig. 1. Proposed link geometry for the train in the curve.

\section{Outage Probability and Average BER}

We consider a OWC system (see Fig. 1) of the groundto-train link along the curved track, using an intensitymodulation/direct detection with on-off keying, which is widely deployed in commercial systems. The laser beams propagate along a horizontal path near ground through a turbulence channel with additive white Gaussian noise. The channel is assumed to be memoryless, stationary, and ergodic, with independent and identically distributed intensity fast fading statistics. The receiver integrates the photocurrent signal which is related to the incident optical power by the detector responsivity for each bit period. In this case, the statistical channel model is given by [8]

$$
y=\eta_{\mathrm{f}} \eta_{\mathrm{r}} \eta_{\mathrm{p}} x+n
$$

where $y$ is the signal at the receiver, $x$ is the modulated signal (and takes values 0 or 1), and $n$ is the additive white Gaussian noise with zero mean and standard deviation $\sigma_{\mathrm{n}} ; \eta_{\mathrm{f}}$ is the random fading due to fluctuation which is caused by atmospheric turbulence and rainfall, $\eta_{\mathrm{r}}$ is the path loss due to the rainfall and is deterministic, and $\eta_{\mathrm{p}}$ is the random attenuation due to pointing errors between the optical transceivers.

\subsection{Distribution of Irradiance Fluctuation}

Take into account the rainfall, the turbulence-induced fluctuation can be modeled as inverse Gaussian distribution [9], [10]. So we model the fading of OWC link with rainfall and atmospheric turbulence as the inverse Gaussian distribution

$$
p\left(\eta_{\mathrm{f}}\right)=\sqrt{\frac{1}{2 \pi \delta_{\mathrm{I}}^{2} \eta_{\mathrm{f}}^{3}}} \exp \left(\frac{1}{\delta_{\mathrm{I}}^{2}}\right) \exp \left(-\frac{\eta_{\mathrm{f}}}{2 \delta_{\mathrm{I}}^{2}}-\frac{\eta_{\mathrm{f}}^{-1}}{2 \delta_{\mathrm{I}}^{2}}\right)
$$

where $\delta_{\mathrm{I}}^{2} \approx \delta_{\mathrm{r}}^{2}+\delta_{\mathrm{a}}^{2}, \delta_{\mathrm{r}}^{2}$ denotes the standard deviation of the intensity rooted in rainfall, and can be given by [12]

$$
\delta_{\mathrm{r}}^{2}=33.32 r_{\mathrm{a}}^{-0.33}-34.22 r_{\mathrm{a}}^{-0.35},
$$

$\delta_{\mathrm{a}}^{2}$ denotes the scintillation index of the atmospheric turbulence for Gaussian-Schell model and untracked beam, and can be given by [5]

$$
\begin{aligned}
\delta_{\mathrm{a}}^{2}\left(\delta_{\mathrm{pe}}, z\right) & \approx \frac{4.42 \delta_{\mathrm{pe}}^{2} \delta^{2}\left[\xi_{\mathrm{s}} z_{0} /\left(r_{0}^{2}+\xi_{\mathrm{s}} z_{0}^{2}\right)\right]^{\alpha / 2-1}}{w_{\mathrm{LT}}^{2}}+3.86 \delta^{2} \\
& \times\left\{0.4 \cos \left[\frac{\alpha-2}{2} \tan ^{-1}\left[\frac{\left(1+2 r_{\mathrm{e}}\right)\left(r_{0}^{2}+\xi_{\mathrm{s}} z_{0}^{2}\right)}{2 \xi_{\mathrm{s}} z_{0}}\right]\right]\right. \\
& \times\left[\left(1+2 r_{\mathrm{e}}\right)^{2}+2\left[\xi_{\mathrm{s}} z_{0} /\left(r_{0}^{2}+\xi_{\mathrm{s}} z_{0}^{2}\right)\right]^{2}\right]^{(\alpha-2) / 4} \\
& \left.-\frac{\alpha}{2 \alpha-2}\left[\xi_{\mathrm{s}} z_{0} /\left(r_{0}^{2}+\xi_{\mathrm{s}} z_{0}^{2}\right)\right]^{\alpha / 2-1}\right\}
\end{aligned}
$$

where $z_{0}=2 z /\left(k w_{0}^{2}\right), \quad w_{0}$ is the effective beam radius at the transmitter, $k=2 \pi / \lambda$ is the optical 
wave number, $r_{0}=1-z / F_{0}, F_{0}$ is the phase curvature parameter of the Gaussian-Schell beam at the transmitter; $r_{\mathrm{e}}=r_{0} /\left\{r_{0}^{2}+\xi_{\mathrm{s}}\left[\xi_{\mathrm{s}} z_{0} /\left(r_{0}^{2}+\xi_{\mathrm{s}} z_{0}^{2}\right)\right]\right\}, \quad \xi_{\mathrm{s}}=$ $1+2 w_{0}^{2} / \rho_{\mathrm{s}}^{2}$ is the source coherence parameter, $\rho_{\mathrm{s}}$ is the spatial coherence length of the source; $\delta^{2}=$ $-8 \pi^{2} A(\alpha) \Gamma(1-\alpha / 2) \sin (\pi \alpha / 4) \tilde{C}_{n}^{2} k^{3-\alpha / 2} z^{\alpha / 2} / \alpha$ is the Rytov variance for plane wave in non-Kolmogorov weak turbulence [6], $A(\alpha)=\Gamma(\alpha-1) \cos (\alpha \pi / 2) /\left(4 \pi^{2}\right), \alpha$ denotes the turbulent spectral index, $\Gamma(x)$ denotes the Gamma function, $\tilde{C}_{n}^{2}=0.033 C_{n}^{2}(k / z)^{(\alpha-11 / 3) / 2} / A(\alpha)$ is the generalized refractive-index structure parameter of turbulent atmosphere with units $\mathrm{m}^{-\alpha+11 / 3}, C_{n}^{2}$ denotes the strength of atmospheric turbulence, and the non-Kolmogorov spectrum is given by [8]

$$
\phi_{n}(\alpha, \kappa)=A(\alpha) \tilde{C}_{n}^{2} \kappa^{-\alpha}, \quad 0 \leq \kappa<\infty, \quad 3<\alpha<5
$$

where $\kappa$ denotes the spatial wave number; $\delta_{\mathrm{pe}}^{2}$ represents variance of pointing errors caused by beam wander, and is given by

$$
\begin{aligned}
\delta_{\mathrm{pe}}^{2} & \approx 219.6 A(\alpha) \tilde{C}_{n}^{2} z^{3} w_{0}^{-1 / 3} \\
\times & \int_{0}^{1}\left\{\frac{\xi^{2}}{\left|\left(1-z / F_{0}\right)+z \xi / F_{0}\right|^{(4-\alpha)}}\right. \\
& \left.-\xi^{2}\left[\frac{\left(2 \pi / r_{\alpha 0}\right)^{2} w_{0}^{2}}{1+\left(2 \pi / r_{\alpha 0}\right)^{2} w_{0}^{2}\left(1-z / F_{0}+z \xi / F_{0}\right)^{2}}\right]^{(2-\alpha / 2)}\right\} \mathrm{d} \xi
\end{aligned}
$$

where $r_{\alpha 0}$ is the Fried's coherence diameter in nonKolmogorov turbulence, which is defined as [5]

$$
r_{\alpha 0}=\left\{\frac{2\left[\frac{8}{\alpha-2} \Gamma\left(\frac{2}{\alpha-2}\right)\right]^{\frac{\alpha-2}{2}}(\alpha-1) \Gamma\left(\frac{3-\alpha}{2}\right)}{\pi^{1 / 2} k^{2} \Gamma\left(\frac{2-\alpha}{2}\right) z \tilde{C}_{n}^{2}\left(\varepsilon+0.62 \Lambda^{\alpha / 2}\right)}\right\}^{1 /(\alpha-2)}
$$

where $\varepsilon=\left(1-\Theta^{\alpha-1}\right) /(1-\Theta)(\Theta \geq 0)$,

$$
\Lambda=\frac{\sqrt{R^{2} \sin ^{2} \omega+\left[d_{1}+R(\cos \omega-1)\right]^{2}} /\left(2 k w_{0}^{2}\right)}{1+\left\{\sqrt{R^{2} \sin ^{2} \omega+\left[d_{1}+R(\cos \omega-1)\right]^{2}} /\left(k w_{0}^{2}\right)\right\}^{2}},
$$

and

$$
\Theta=\frac{1}{2}\left\{1+\frac{R^{2} \sin ^{2} \omega+\left[d_{1}+R(\cos \omega-1)\right]^{2}}{k^{2} w_{0}^{4}}\right\}^{-1} .
$$

\subsection{Raining Attenuation}

Under the rainy weather, the attenuation induced by the rainwater absorption through the channel leads to the path loss. The attenuation of laser power through the channel is described by the exponential Beers-Lambert Law as [8]

$$
\eta_{\mathrm{r}}=\exp \left[-\chi \sqrt{R^{2} \sin ^{2} \omega+\left(d_{1}+R \cos \omega-R\right)^{2}}\right]
$$

where $\chi$ denotes the raining attenuation coefficient. For the ITU-France model, it can be written as [13]

$$
\chi=1.076 r_{\mathrm{a}}{ }^{0.67}, \quad[\mathrm{~dB} / \mathrm{km}]
$$

where $r_{\mathrm{a}}$ is the rainfall intensity with unit $\mathrm{mm} / \mathrm{h}$.

\subsection{Channel State Distribution with Pointing Errors}

For the ground-to-train communication link, the vibration of the train causes the misalignment of communication beams and signal fading at the receiver. The probability distribution function of pointing errors $\eta_{\mathrm{p}}$ considering independent identical Gaussian distributions can be expressed as [14]

$$
p\left(\eta_{\mathrm{p}}\right)=\frac{\gamma^{2} \eta_{\mathrm{p}}^{\gamma^{2}-1}}{M_{0}^{\gamma^{2}}}, \quad 0 \leq \eta_{\mathrm{p}} \leq M_{0}
$$

where $\gamma=w_{\mathrm{zeq}} / 2 \sigma_{\mathrm{s}}$ is the ratio between the equivalent beam radius at the receiver and the pointing errors standard deviation $\sigma_{\mathrm{s}}$ at the receiver, $M_{0}=[\operatorname{erf}(v)]^{2}$ is the fraction of the received power at a zero radial distance, $v=\sqrt{\pi} a /\left(\sqrt{2} w_{\mathrm{LT}}\right)$, and $a$ is the radius of the circular detection aperture, $w_{\text {zeq }}$ is the equivalent beam width, which is defined as

$$
w_{\mathrm{zeq}}^{2}=w_{\mathrm{LT}}^{2} \sqrt{\pi} \operatorname{erf}(v)\left[2 v \exp \left(-v^{2}\right)\right]^{-1}
$$

where $w_{\mathrm{LT}}^{2}=w_{0}^{2}\left[r_{0}^{2}+\left(\xi_{\mathrm{s}}+\frac{2 w_{0}^{2}}{\rho_{0}^{2}(z, \alpha)}\right)\left(\frac{\lambda z}{\pi w_{0}^{2}}\right)^{2}\right]$ is the effective beam spot radius at the receiver [9], $\rho_{0}^{2}(z, \alpha)=$ $\left[-2^{3-\alpha} \pi^{2} \Gamma(1-\alpha / 2) k^{2} A(\alpha) \tilde{C}_{n}^{2} z / \Gamma(\alpha / 2)\right]^{-1 /(\alpha-2)}$ is the spatial coherence radius of a spherical wave propagating in turbulence [15].

The probability distribution of $\eta=\eta_{\mathrm{r}} \eta_{\mathrm{f}} \eta_{\mathrm{p}}$ can be expressed as [8]

$$
p\left(\eta, w_{\mathrm{LT}}\right)=\int p\left(\eta / \eta_{\mathrm{f}}\right) p\left(\eta_{\mathrm{f}}\right) \mathrm{d} \eta_{\mathrm{f}}
$$

where $p\left(\eta, w_{\mathrm{LT}}\right)$ denotes a family of probability density function parameterized by the beam width $w_{\mathrm{LT}}$, and $p\left(\eta / \eta_{\mathrm{f}}\right)$ is the conditional probability given a turbulence state $\eta_{\mathrm{f}}$.

For the inverse Gaussian distribution, recall that $\eta_{\mathrm{r}}$ is deterministic and acts as a scaling factor, the channel state distribution with pointing errors can be expressed by

$$
\begin{aligned}
p\left(\eta, w_{\mathrm{LT}}\right)= & \frac{\gamma^{2} \eta^{\gamma^{2}-1}}{\sqrt{2 \pi \delta_{\mathrm{I}}^{2}}\left(M_{0} \eta_{\mathrm{r}}\right)^{\gamma^{2}}} \exp \left(\frac{1}{\delta_{\mathrm{I}}^{2}}\right) \int_{\eta / M_{0} \eta_{\mathrm{r}}}^{1} \eta_{\mathrm{f}}^{-\gamma^{2}-3 / 2} \\
& \times \exp \left(-\frac{\eta_{\mathrm{f}}}{2 \delta_{\mathrm{I}}^{2}}-\frac{\eta_{\mathrm{f}}^{-1}}{2 \delta_{\mathrm{I}}^{2}}\right) \mathrm{d} \eta_{\mathrm{f}} .
\end{aligned}
$$

By the generating function of the modified Bessel function $\mathrm{I}_{m}(\cdot)$ [16], i.e.,

$$
\exp \left[\frac{x}{2}\left(z+z^{-1}\right)\right]=\sum_{m=-\infty}^{\infty} \mathrm{I}_{m}(x) z^{m}, \quad(z \neq 0),
$$

we have 


$$
\begin{aligned}
p\left(\eta, w_{\mathrm{LT}}\right)= & \frac{\gamma^{2} \eta^{\gamma^{2}-1}}{\sqrt{2 \pi \delta_{\mathrm{I}}^{2}}\left(M_{0} \eta_{\mathrm{r}}\right)^{\gamma^{2}}} \exp \left(\frac{1}{\delta_{\mathrm{I}}^{2}}\right) \sum_{m=-\infty}^{\infty} \mathrm{I}_{m}\left(-\frac{1}{\delta_{\mathrm{I}}^{2}}\right) \\
& \times \frac{1}{m-\gamma^{2}-1 / 2}\left[1-\left(\frac{\eta}{M_{0} \eta_{\mathrm{r}}}\right)^{m-\gamma^{2}-1 / 2}\right] .
\end{aligned}
$$

Under the combined influence of rainfall-induced pass loss, raining turbulence attenuation, and pointing errors loss, considering a far field propagation in square approximation of the phase structure function, the received power at the receiver along the z-axis for a Gaussian-Schell beam is given by [4]

$$
P_{\mathrm{r}}=\eta_{\mathrm{r}} \cdot 2 P_{\mathrm{t}} \cdot\left(\frac{2 a}{w_{\text {zeq }}}\right)^{2}\left[\operatorname{erf}\left(\frac{\sqrt{\pi} a}{\sqrt{2} w_{\mathrm{LT}}}\right)\right]^{2} \exp \left(-\frac{2 r^{2}}{w_{\text {zeq }}^{2}}\right)
$$

where $P_{\mathrm{t}}$ is the total transmit power from station.

\subsection{Outage Probability}

Outage probability is a statistical measure for the quality of a fading channel, and generally defined as the percentage of time that the instantaneous quality of the channel is below a satisfactory threshold. We assume the transmitter sends information at a rate of $R_{0}$ bits/channel use, then the outage probability is the finite probability that the instantaneous capacity is not sufficient to support $R_{0}$ [14].

The outage probability of the inverse Gaussian turbulence channel is given by

$$
\begin{aligned}
p_{\text {out }}= & \int_{0}^{\eta_{0}} p\left(\eta, w_{\mathrm{LT}}\right) \mathrm{d} \eta \\
= & \frac{\gamma^{2}}{\sqrt{2 \pi \delta_{\mathrm{I}}^{2}}\left(M_{0} \eta_{\mathrm{r}}\right)^{\gamma^{2}}} \exp \left(\frac{1}{\delta_{\mathrm{I}}^{2}}\right) \sum_{m=-\infty}^{\infty} \mathrm{I}_{m}\left(-\frac{1}{\delta_{\mathrm{I}}^{2}}\right) \\
& \times \frac{1}{m-\gamma^{2}-1 / 2} \int_{0}^{\eta_{0}}\left[\eta^{\gamma^{2}-1}-\frac{\eta^{m-3 / 2}}{\left(M_{0} \eta_{\mathrm{r}}\right)^{m-\gamma^{2}-1 / 2}}\right] \mathrm{d} \eta \\
= & \frac{\gamma^{2}}{\sqrt{2 \pi \delta_{\mathrm{I}}^{2}}} \exp \left(\frac{1}{\delta_{\mathrm{I}}^{2}}\right) \sum_{m=-\infty}^{\infty} \frac{1}{m-\gamma^{2}-1 / 2} \mathrm{I}_{m}\left(-\frac{1}{\delta_{\mathrm{I}}^{2}}\right) \\
& \times\left[\frac{1}{\gamma^{2}}\left(\frac{\eta_{0}}{M_{0} \eta_{\mathrm{r}}}\right)^{\gamma^{2}}-\frac{1}{m-1 / 2}\left(\frac{\eta_{0}}{M_{0} \eta_{\mathrm{r}}}\right)^{m-1 / 2}\right]
\end{aligned}
$$

where $\eta_{0}$ denotes the threshold value of instantaneous intensity gain.

\subsection{Average BER}

BER is the indicator that estimates the accuracy of the data transmission. The BER of intensity- modulation/direct detection with on-off keying is given by

$$
P_{\mathrm{b}}(e)=p(1) p(e \mid 1)+p(0) p(e \mid 0)
$$

where $p(1)$ and $p(0)$ are the probabilities of sending 1 and 0 bits, respectively; $p(e \mid 1)$ and $p(e \mid 0)$ denote the conditional bit error probabilities when the transmitted bit is 1 and 0 , respectively. Considering $p(1)=p(0)=1 / 2$ and $p(e \mid 1)=p(e \mid 0)$, we have conditioned on $\eta[8]$.

$$
P_{\mathrm{b}}(e \mid \eta)=p(e \mid 1, \eta)=(e \mid 0, \eta)=\frac{1}{2} \operatorname{erfc}\left(\frac{P_{\mathrm{t}} \eta}{\sigma_{n}}\right) .
$$

The average BER in the present of raining turbulence and pointing errors can be obtained by

$$
\begin{aligned}
& P_{\mathrm{BER}}(e)=\int_{0}^{\infty} P_{\mathrm{b}}(e \mid \eta) p\left(\eta, w_{\mathrm{LT}}\right) \mathrm{d} \eta \\
& =\frac{\gamma^{2}}{2 \sqrt{2 \pi \delta_{\mathrm{I}}^{2}}\left(M_{0} \eta_{\mathrm{r}}\right)^{\gamma^{2}}} \exp \left(\frac{1}{\delta_{\mathrm{I}}^{2}}\right) \sum_{m=-\infty}^{\infty} \mathrm{I}_{m}\left(-\frac{1}{\delta_{\mathrm{I}}^{2}}\right) \frac{1}{m-\gamma^{2}-1 / 2} \\
& \quad \times \int_{0}^{\infty} \eta^{\gamma^{2}-1}\left[1-\left(\frac{\eta}{M_{0} \eta_{\mathrm{r}}}\right)^{m-\gamma^{2}-1 / 2}\right] \operatorname{erfc}\left(\frac{P_{\mathrm{t}} \eta}{\sigma_{n}}\right) \mathrm{d} \eta .
\end{aligned}
$$

Expressing the $\operatorname{erfc}(x)$ as Meijer's G-functions i.e., $\operatorname{erfc}(x)=\frac{1}{\sqrt{\pi}} \mathrm{G}_{1,2}^{2,0}\left(x^{2} \mid \begin{array}{c}1 \\ 0,1 / 2\end{array}\right)$ [16], we have a series solution to BER

$$
\begin{aligned}
& P_{\mathrm{BER}}(e)=\frac{\gamma^{2}}{4 \sqrt{2} \pi \delta_{\mathrm{I}}\left(M_{0} \eta_{\mathrm{r}}\right)^{\gamma^{2}}} \exp \left(\frac{1}{\delta_{\mathrm{I}}^{2}}\right) \sum_{m=-\infty}^{\infty} \mathrm{I}_{m}\left(-\frac{1}{\delta_{\mathrm{I}}^{2}}\right) \\
& \times \frac{1}{m-\gamma^{2}-1 / 2}\left[\mathrm{G}_{3,4}^{2,2}\left(\frac{P_{\mathrm{t}}^{2}}{\sigma_{n}^{2}} \mid \begin{array}{c}
\frac{1-\gamma^{2}}{2}, \frac{2-\gamma^{2}}{2}, 1 \\
0, \frac{1}{2},-\frac{\gamma^{2}}{2}, \frac{1-\gamma^{2}}{2}
\end{array}\right)\right. \\
&\left.-\left(M_{0} \eta_{\mathrm{r}}\right)^{\gamma^{2}+1 / 2-m} \mathrm{G}_{3,4}^{2,2}\left(\frac{P_{\mathrm{t}}^{2}}{\sigma_{n}^{2}} \mid \begin{array}{c}
\frac{3}{4}-\frac{m}{2}, \frac{5}{4}-\frac{m}{2}, 1 \\
0, \frac{1}{4}-\frac{m}{2}, \frac{3}{4}-\frac{m}{2}
\end{array}\right)\right] .
\end{aligned}
$$

\section{Numerical Results}

In this section, we discuss the performance of the ground-to-train link of the curved track in rainy weather, in terms of the received power, outage probability, and average BER. We present numerical results for Gaussian Schellmodel and untracked beam propagation along an atmosphere turbulence channel with pointing errors in rainy weather in Fig. 2-11. We assume a OWC system with the vertical separation of $d_{1}=1 \mathrm{~m}$, the horizontal separation of $d_{2}=15 \mathrm{~m}$, the noise standard deviation of $\sigma_{\mathrm{n}}=10^{-7} \mathrm{~A} / \mathrm{Hz}$, the threshold value of instantaneous intensity gain of $\eta_{0}=3.08 \times 10^{-5}$, the receive aperture of size $2 a=20 \mathrm{~cm}$, the phase curvature parameter of the beam of $F_{0}=-z$, and the average transmitted optical power of $P_{\mathrm{t}}=25 \mathrm{~mW}$.

To highlight the influence of the beam wander and the strength of atmospheric turbulence on the received signal power, we depict the curves of the variance of pointing errors and the strength of atmospheric turbulence in Fig. 2. The parameters of link are given by $R=300 \mathrm{~m}, \lambda=1550 \mathrm{~nm}$, $\alpha=11 / 3, w_{0} / a=30, r_{\mathrm{a}}=5 \mathrm{~mm} / \mathrm{h}$, and $\rho_{\mathrm{s}}=0.02 \mathrm{~m}$. The larger variance of the pointing errors obtains the worse received signal power, since the Gaussian-Schell beam center deviates from the receiver which increases the loss of the 


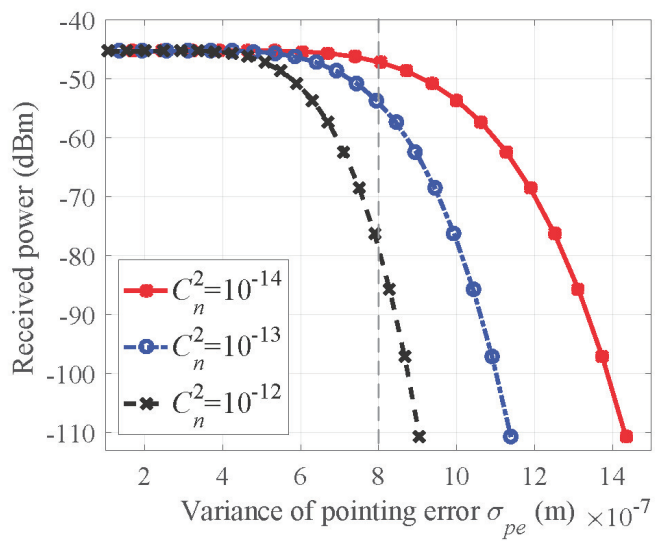

Fig. 2. Received power of the link as a function of the variance of pointing errors caused by beam wander for different strength of atmospheric turbulence.

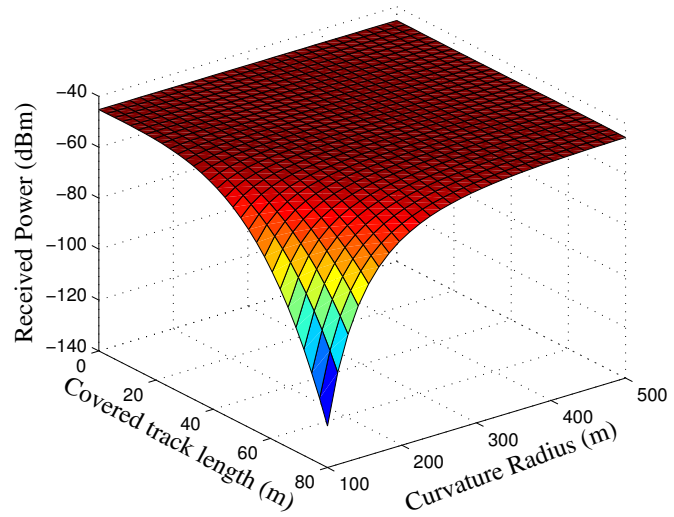

Fig. 3. Received power as a function of the covered track length and curvature radius of the curved track.

propagation beams. In addition, we notice that, the strength of atmospheric turbulence has a little impact on the received power when $\sigma_{\mathrm{pe}}<5.5 \times 10^{-7}$. However, for instant, at the variance of pointing error of $8 \times 10^{-7} \mathrm{~m}$, the received power value reduced to $-80 \mathrm{dBm}$ under the strong turbulence, while under the weak turbulence it is $-47 \mathrm{dBm}$. We know that, the variance of pointing errors is proportional to the transmission distance and the strength of turbulence [5], therefore, for the fixed value of $\sigma_{\mathrm{pe}}$, the transmission distance should be shorter when the strength of turbulence increases. So Fig. 2 shows the impact of the strength of atmospheric turbulence is stronger than that of the cover track length in the OWC link, according to the received power.

To select a suitable value of the curvature radius for our calculation, we simulate the effect of $R$ on the received power of the link in Fig. 3. The parameters are as $\lambda=1550 \mathrm{~nm}$, $\alpha=11 / 3, w_{0} / a=30, r_{\mathrm{a}}=5 \mathrm{~mm} / \mathrm{h}, \rho_{\mathrm{s}}=0.02 \mathrm{~m}$, and $C_{n}^{2}=1 \times 10^{-13} \mathrm{~m}^{-2 / 3}$. For the rail track, the typical bend could have curvature radius of $200-500 \mathrm{~m}$. However, the bends can be sharper in some cases where $R$ might be as small as $100 \mathrm{~m}$ [7]. So, the curvature radius $R$ varies from 100 to $500 \mathrm{~m}$ in our numerical calculation. Figure 3 shows that the received power fades with the increase of the covered track length, but the smaller $R$ makes the received power lower. As the worst case scenario, we have assumed that $R=100 \mathrm{~m}$ in the following numerical analysis.

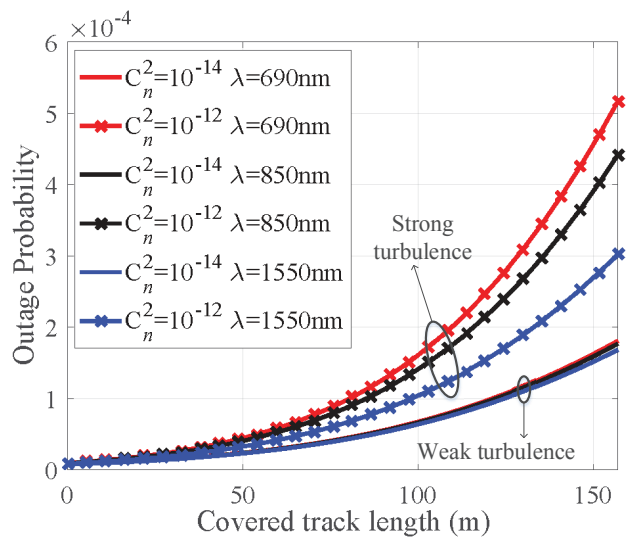

Fig. 4. Outage probability as a function of the covered track length for different strength of atmospheric turbulence and operating wavelength.

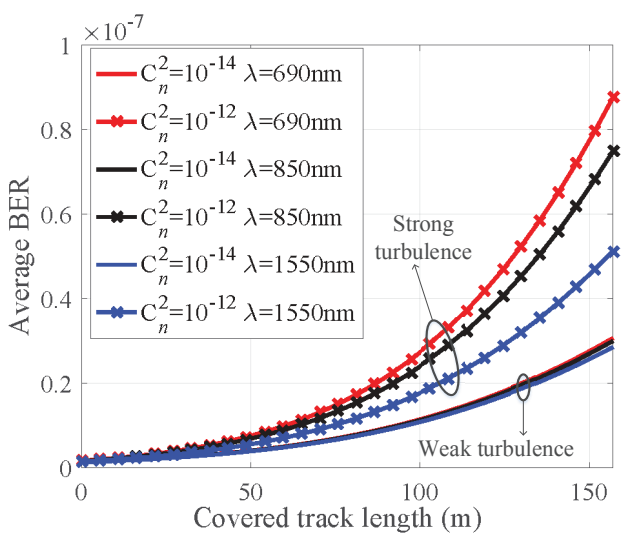

Fig. 5. Average BER as a function of the covered track length for different strength of atmospheric turbulence and operating wavelength.

Figure 4 and 5 present the performance for different strength of atmospheric turbulence $C_{n}^{2}$ and operating wavelength $\lambda$ at different covered track length $L$. The length of the curved track for $R=100 \mathrm{~m}$ is $157 \mathrm{~m}$, thus we select $157 \mathrm{~m}$ as the maximum number of $L$ in our calculation. The parameters are given by $\alpha=11 / 3, \sigma_{\mathrm{s}} / a=3, w_{0} / a=5$, $r_{\mathrm{a}}=20 \mathrm{~mm} / \mathrm{h}$, and $\rho_{\mathrm{s}}=0.02 \mathrm{~m}$. We notice that the outage probability and average BER both raise with the increase of the covered track length. The change of the outage probability and average BER for the strong turbulence is obvious, but for the weak turbulence, the change is small. It implies that the strong turbulence scenario damage the stability and accuracy of the data transmission, and the atmospheric turbulence has greater impact than the cover track length on the OWC link, which is consistent with the consequence of Fig. 2. For different cover track length, the strong turbulence of $C_{n}^{2}=1 \times 10^{-12} \mathrm{~m}^{-2 / 3}$ combined with the short operating wavelength of $\lambda=690 \mathrm{~nm}$ achieves the highest value of outage probability and average BER. Thus we can use long wavelength of the signal source to obtain better performance of the system in the strong turbulence channel, and ignore the effect of the change of the wavelength for the weak turbulence channel. 


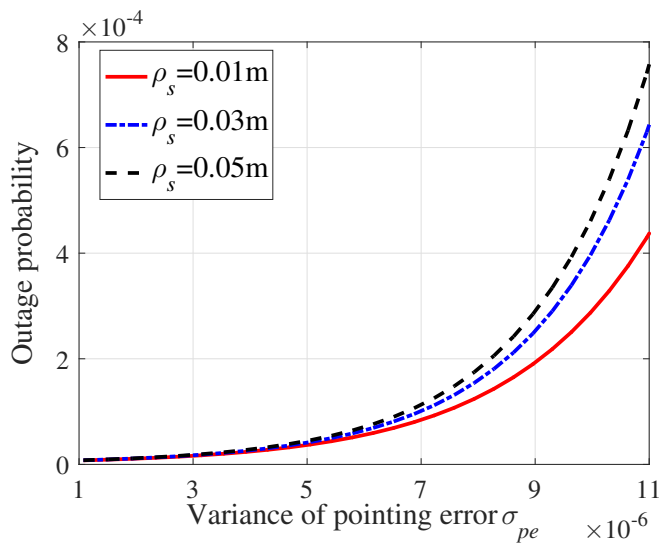

Fig. 6. Outage probability as a function of the variance of pointing errors caused by beam wander for different spatial coherence length.

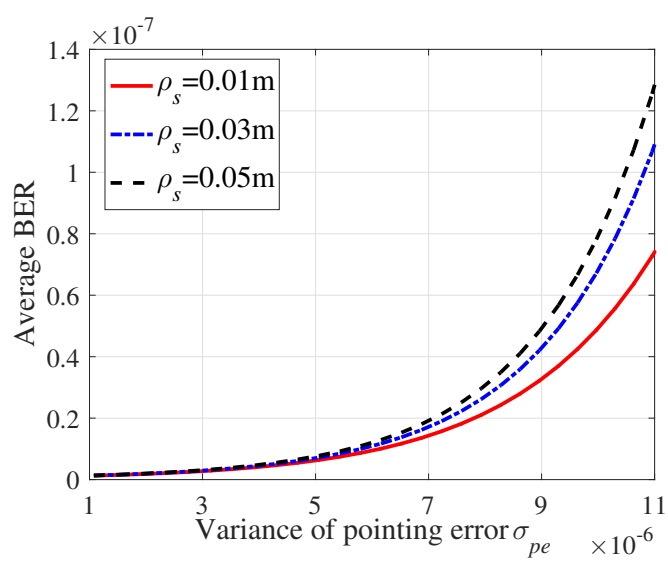

Fig. 7. Average BER as a function of the variance of pointing errors caused by beam wander for different spatial coherence length.

To demonstrate the effect of the coherence of partial coherence beams and beam wander-induced pointing errors on the system performance, we plot the curves of the outage probability and average BER as a function of the variance of pointing errors $\sigma_{\text {pe }}$ for different spatial coherence length $\rho_{\mathrm{s}}$ in Fig. 6 and 7 . The system parameters are given by $L=75 \mathrm{~m}, \lambda=1550 \mathrm{~nm}, C_{n}^{2}=1 \times 10^{-13} \mathrm{~m}^{-2 / 3}, \alpha=11 / 3$, $\sigma_{\mathrm{s}} / a=3, w_{0} / a=5$, and $r_{\mathrm{a}}=20 \mathrm{~mm} / \mathrm{h}$. The larger variance of pointing errors improves the intensity fading, the irradiance fluctuation, and the noise of the channel, consequently, the performance of the link get worse according to the high level of the outage probability and average BER in Fig. 6 and 7. Besides, we observe that, the longer the spatial coherence length is, the higher the outage probability and average BER of the ground-to-train channel are. The phenomenon is triggered by that, the coherence of partial coherence beams enhances the scintillation of the signal, which can increase the optical intensity fluctuation and the noise of channel. Therefore, channels with partial coherent beams have lower stability and accuracy.

We present the impact of the beam width normalized by the radius of the receiver aperture together with the normalized jitter in Fig. 8 and 9. The system parameters of the link

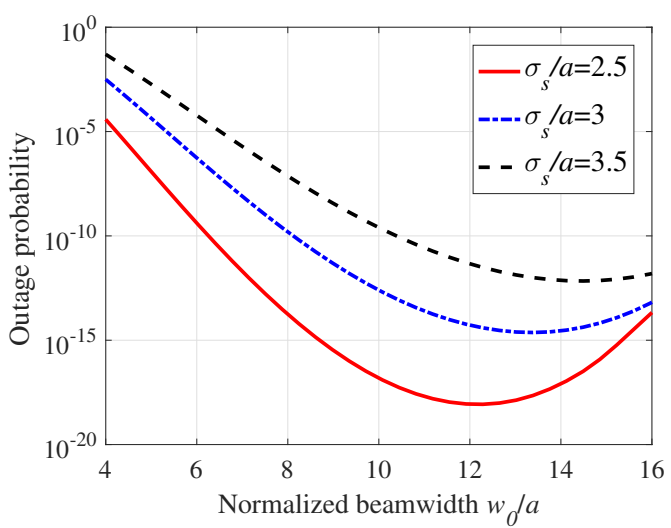

Fig. 8. Outage probability as a function of the normalized beam width for different normalized jitter.

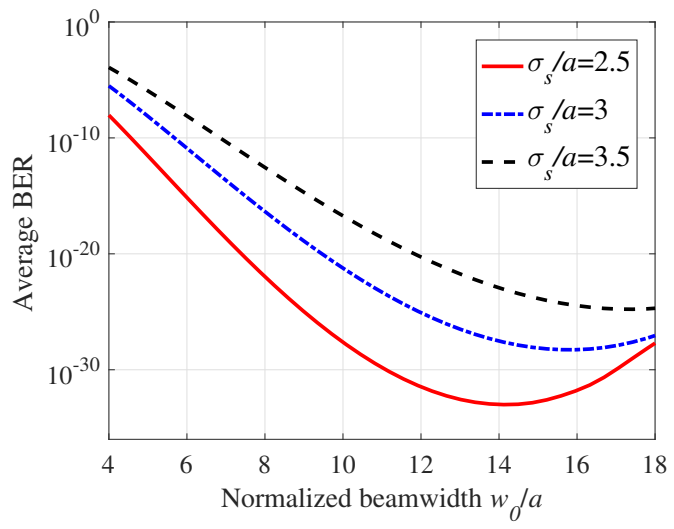

Fig. 9. Average BER as a function of the normalized beam width for different normalized jitter.

are given by $L=75 \mathrm{~m}, \lambda=1550 \mathrm{~nm}, C_{n}^{2}=1 \times 10^{-13} \mathrm{~m}^{-2 / 3}$, $\alpha=11 / 3, r_{\mathrm{a}}=20 \mathrm{~mm} / \mathrm{h}$, and $\rho_{\mathrm{s}}=0.02 \mathrm{~m}$. Figure 8 and 9 show that for given the receiver aperture, there is a optimum emitting beam width and the optimum emitting beam width increases with the increasing of the normalized jitter. This result indicates that we can choose the size of transmitting and receiving apertures to optimize the performance of the OWC link. Figure 8 and 9 also show that the pointing errors due to the misalignment of the communication beams will increase the intensity fading, the irradiance fluctuation, and the noise of channel, thereby resulting in higher outage probability and average BER of the OWC link. Overall, by improving the shockproof performance of the tracks, we can enhance the communication quality of the OWC link.

To understand the effect of the rainfall and topography, we depict the outage probability and average BER of the channel as a function of the rainfall intensity $r_{\mathrm{a}}$ for different non-Kolmogorov spectral index $\alpha$ in Fig. 10 and 11. The system parameters are given by $L=75 \mathrm{~m}, \lambda=1550 \mathrm{~nm}$, $C_{n}^{2}=1 \times 10^{-13} \mathrm{~m}^{-2 / 3}, \sigma_{\mathrm{s}} / a=3, w_{0} / a=8$, and $\rho_{\mathrm{s}}=0.02 \mathrm{~m}$. In order to show the effect of the non-Kolmogorov spectral index more obviously, we enlarged the curves at about $r_{\mathrm{a}}=100 \mathrm{~mm} / \mathrm{h}$. The curves in Fig. 10 and 11 raise with the increase of the rainfall intensity, and the value of them reach a relatively high magnitude after $\left(r_{\mathrm{a}}>100 \mathrm{~mm} / \mathrm{h}\right)$. It implies that the outage probability and average BER increase 


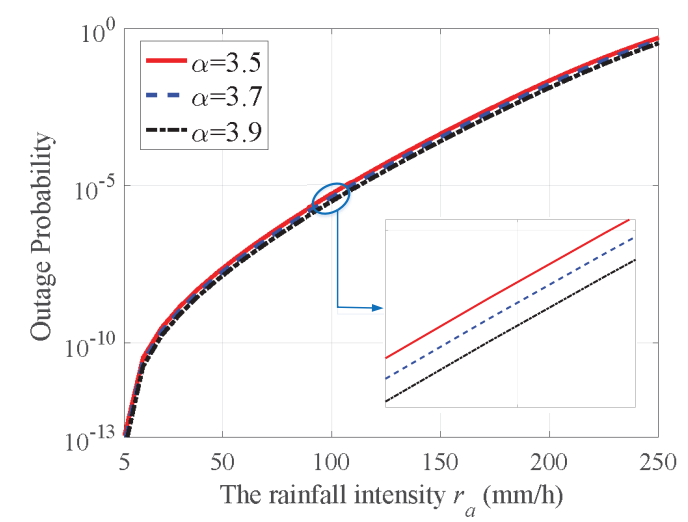

Fig. 10. Outage probability as a function of the rainfall intensity for different non-Kolmogorov spectral index.

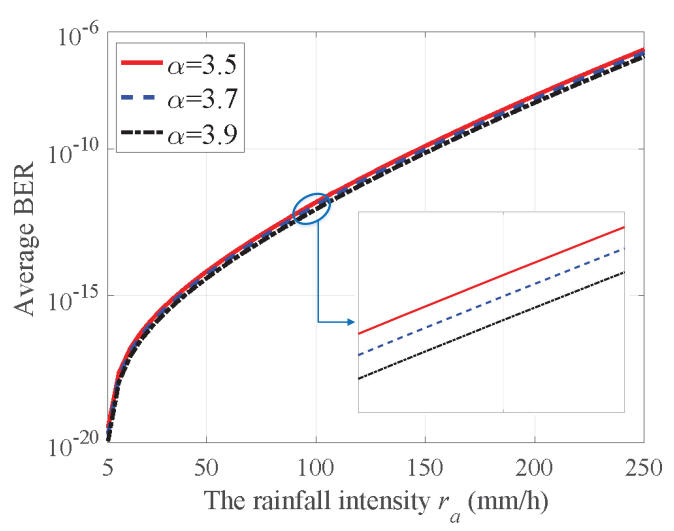

Fig. 11. Average BER as a function of the rainfall intensity for different non-Kolmogorov spectral index.

with the heavier rain for the rest parameters being same. The performance of the OWC link in rainstorm weather $\left(50 \mathrm{~mm} / \mathrm{h}<\mathrm{r}_{\mathrm{a}}<100 \mathrm{~mm} / \mathrm{h}\right)$ is poor, especially in heavy rainstorm $\left(r_{\mathrm{a}}>100 \mathrm{~mm} / \mathrm{h}\right)$. Moreover, for different rainfall intensity, the outage probability and average BER reach a lower value with the non-Kolmogorov spectral index approaching to 4 . The non-Kolmogorov spectral index, which varies with the topography, can influence the extent of the optical signal scintillation, hence, it degrades the outage probability and average BER of the link. As a consequence, we should consider the complex topography where the train passes as well.

\section{Conclusions}

In this work, using the inverse Gaussian distribution models of the raining turbulence, we presented mathematical expressions for the evaluation of outage probability and the average BER of OWC links for the ground-to-train of the curved track with pointing errors. Our results illustrate that, in raining weather, the designer of the ground-to-train system should take account of the dominant factors - pointing errors induced by beam wander and train vibration. The rainfall can disrupt the quality of the OWC link, especially the rainstorm. The improving of the shockproof performance of the tracks and using long wavelength of the signal source will improve the communication performance of OWC links.
Moreover, we can choose the size of communication transmitting and receiving apertures to optimize the performance of the OWC link. The atmospheric turbulence has greater impact than the cover track length on the OWC link.

\section{Acknowledgments}

This work was supported by the Fundamental Research Funds for the Central Universities (Grant No. JUSRP51716A).

\section{References}

[1] LIU, Q., QIAO, C., MITCHELL, G., et al. Optical wireless communication networks for first- and last-mile broadband access. [Invited] Journal of Optical Networking, 2005, vol. 4, no. 1, p. 807-828. DOI: $10.1364 / \mathrm{JON} .4 .000807$

[2] GARCÍA-ZAMBRANA, A., CASTILLO-VÁZQUEZ, B., CASTILLO-VÁZQUEZ, C. Asymptotic error-rate analysis of FSO links using transmit laser selection over gamma-gamma atmospheric turbulence channels with pointing errors. Optics Express, 2012, vol. 20, no. 3, p. 2096-2109. DOI: 10.1364/OE.20.002096

[3] EL-WAKEEL, A. S., MOHAMMED, N. A., ALY, M. H. Free space optical communications system performance under atmospheric scattering and turbulence for 850 and $1550 \mathrm{~nm}$ operation. Applied Optics, 2016, vol. 55, no. 26, p. 7276-7286. DOI: 10.1364/AO.55.007276

[4] PAUDEL, R., GHASSEMLOOY, Z., LE-MINH, H., et al. Modelling of free space optical link for ground-to-train communications using a Gaussian source. IET Optoelectronics, 2013, vol. 7, no. 1, p. 1-8. DOI: 10.1049/iet-opt.2012.0047

[5] ANDREWS, L. C., PHILliPS, R. L. Laser Beam Propagation through Random Media. 2nd ed., New York: SPIE, 2005. ISBN: 9780819459480

[6] GAO, J., ZHANG, Y., CHENG, M., et al. Average capacity of ground-to-train optical wireless communication links in the nonKolmogorov and gamma-gamma distribution turbulence with pointing errors. Optics Communications, 2016, vol. 358, p. 147-153. DOI: 10.1016/j.optcom.2015.09.035

[7] PAUDEL, R., POLIAK, J., GHASSEMLOOY, Z., et al. Curved track analysis of FSO link for ground-to-train communications. Radioengineering, 2014, vol. 23, no. 1, p. 452-459. ISSN: 1210-2512

[8] YANG, Y., YU, L., ZHANG, Y. Average capacity of the ground to train communication link of a curved track in the turbulence of gamma-gamma distribution. Optics Communications, 2017, vol. 389, p. 68-73. DOI: 10.1016/j.optcom.2016.12.025

[9] CHATZIDIAMANTIS, N. D., SANDALIDIS, H. G., KARAGIANNIDIS, G. K., et al. Inverse Gaussian modeling of turbulence-induced fading in free-space optical systems. Journal of Lightwave Technology, 2011, vol. 29, no. 10, p. 1590-1596. DOI: 10.1109/JLT.2011.2132792

[10] KOUROGiorgas, C. I., PANAgOPOUlos, A. D., KANELLOPOULOS, J. D. A new method for the prediction of outage probability of LOS terrestrial links operating above $10 \mathrm{GHz}$. IEEE Antennas \& Wireless Propagation Letters, 2013, vol. 12, p. 516-519. DOI: 10.1109/LAWP.2013.2254460

[11] ABU-AlmaAlie, Z., GHASSEMLOOY, Z., BHATNAGAR, M. R., et al. Investigation on iterative multiuser detection physical layer network coding in two-way relay free-space optical links with turbulences and pointing errors. Applied Optics, 2016, vol. 55, no. 33, p. 9396-9406. DOI: 10.1364/AO.55.009396 
[12] LUINI, L., CAPSONI, C. Multiexcell: A new rain field model for propagation applications. IEEE Transactions on Antennas \& Propagation, 2011, vol. 59, no. 59, p. 4286-4300. DOI: $10.1109 /$ TAP.2011.2164175

[13] BASAHEL, A., RAFIQUL, I. M., HABAEBI, M. H., et al. A proposed rain attenuation prediction method for free space optical link based on rain rate statistics. ARPN Journal of Engineering and Applied Sciences, 2015, vol. 10, no. 23, p. 17351-17355. ISSN: 18196608

[14] FARID, A. A., HRANILOVIC, S. Outage capacity optimization for free-space optical links with pointing errors. Journal of Lightwave Technology, 2007, vol. 25, no. 7, p. 1702-1710. DOI: 10.1109/JLT.2007.899174

[15] BELAND, R. R. Some aspects of propagation through weak isotropic non-Kolmogorov turbulence. In Proceedings of the SPIE 2375, Beam Control, Diagnostics, Standards and Propagation. 1995, vol. 2375 , p. 6-16. DOI: $10.1117 / 12.206979$

[16] WOLFRAM RESEARCH. Wolfram Functions Site. [Online] Cited: 2017-01-26. Available at: http://functions.wolfram.com
About the Authors ...

Yanqiu YANG was born in Jiangsu province, China. She received the B.S. degree in 2011. She is currently pursuing the M.E. degree in the Jiangnan University. Her research interest is optical wireless communication.

Yixin ZHANG (corresponding author) was born in Jiangsu province, China. He received the Ph.D. degree in Nanjing University of Science and Technology, and has been a professor of Jiangnan University since 1994. He has authored and coauthored more than 200 scientific papers in major journals and international conferences. His research interest is optical wireless communication.

Lin YU was born in Anhui province China. She received the Ph.D. degree in Tianjin University, and has been a lectorate of Jiangnan University since 2016. Her research interest is optical wireless communication. 